\title{
14 Orthopaedic Physical Examination Assisting System for Improvement of Accuracy and Reproducibility of Knee Laxity Diagnosis*
}

\author{
Jun OKAMOTO**, Yu KUMASAKA ${ }^{* * *}$, Kazuya KAWAMURA ${ }^{\dagger}$, \\ Tomoyuki MATSUMOTO $^{\dagger \dagger}$, Seiji KUBO ${ }^{\dagger \dagger}$, Hirotsugu MURATSU ${ }^{\dagger \dagger \dagger}$, \\ Masahiro KUROSAKA ${ }^{\dagger \dagger}$ and Masakatsu G. FUJIE ${ }^{\dagger}$ \\ ** Institute of Advanced Biomedical Engineering \& Science, Tokyo Women's Medical University, \\ 8-1 Kawada-cho, Shinjuku-ku, Tokyo 162-8666, Japan \\ E-mail: j-okamoto@abmes.twmu.ac.jp \\ *** Graduate School of Science \& Engineering, Waseda University, \\ 3-4-1 Ohkubo, Shinjuku-ku, Tokyo 169-5555, Japan \\ $\dagger$ Faculty of Science \& Engineering, Waseda University, \\ 3-4-1 Ohkubo, Shinjuku-ku, Tokyo 169-5555, Japan \\ $\dagger$ Department of Orthopaedic Surgery, Kobe graduate school of medicine, \\ 7-5-2 Kusunoki-cho, Chuo-ku, Kobe-city 650-0017, Japan \\ $\dagger \dagger$ Department of Orthopaedic Surgery, Steel Memorial Hirohata Hospital, \\ 3-1 Yumesaki-cho, Hirohata-ku, Himeji-city 671-1122, Japan
}

\begin{abstract}
In this study, to improve accuracy and reproducibility of knee laxity diagnosis, Orthopaedic physical examination assisting system was developed. The system enables the surgeon to monitor the force vector added by himself and the posture of the knee in real-time. The advantage of the developed system is to quantify the normal orthopaedic physical examination, without any modification, which is accustomed to general orthopaedic surgeons. The force vector added to the shank by a surgeon is measured with 3 axis force sensor and 6DOF magnetic tracker FASTRAK, and 6DOF knee joint motion of the patient is measured with two FASTRAK receivers which are attached on its thigh and shank. In this system, to describe the 6DOF knee motion, Femoral and tibial coordinate systems are defined with bony landmark which is easily palpated and are registered to the thigh or shank receiver coordinate system by homogeneous transformation matrix which is acquired by the special stylus. To evaluate the effectiveness of the system, the posterior drawer test with the system was done by 5 young healthy people. The evaluation parameters were knee posture, load magnitude and load position during the experiment. The result showed examination accuracy was significantly improved in all evaluation parameters.
\end{abstract}

Key words : Medical and Welfare Assistance, Biomechanics, Biomotion, Orthopaedic Physical Examination, Knee Laxity Diagnosis, Magnetic Tracker, Medical Quantitation, Weber Ratio

\section{Introduction}

In orthopaedic diagnosis, the physician manually applies a load to the affected site, and makes a judgment on the disorder and its grade based on the resultant displacement of the bone and range of motion. This procedure is called orthopaedic physical examination. However, because diagnosis in orthopaedic physical examination relies upon the hand of the physician and the reaction of the patient, the results depend on qualitative judgment. This leads to problems such as differences in diagnosis between physicians, adverse effects on treatment due to the improper identification of grade, and non-reproducibility. To address these problems, 
we developed "The Orthopaedic Physical Examination Assisting System," which exhibits the force applied by a physician and the resultant motion of the knee joint for the evaluation of joint laxity in knee ligament injury in real-time as the diagnosis material of the joint laxity, and quantitatively records them. This system strives for accuracy and reproducibility in the evaluation of joint laxity. In this paper, we describe the concept of the system, as well as the results of an evaluation on improvements in the accuracy and reproducibility of orthopaedic physical examination with this system and its training effect.

2. The orthopaedic physical examination assisting method best suited to clinical settings

\subsection{Outline of orthopaedic physical examination in orthopaedics}

As the number of athletes and the elderly is increasing, the number of people seeking orthopaedic consultation is increasing year by year. In particular, clinics are seeing a large number of knee joint patients. In this study, therefore, we focus on joint laxity in the evaluation of ligament injury. Orthopaedic diagnosis begins with inquiry by the physician. This is followed by visual inspection and palpation. Orthopaedic physical examination is the basis of this palpation ${ }^{(1)}$. In orthopaedic physical examination, the physician manually applies stress to the affected site. The resultant displacement of the bone, and reduction phenomenon are evaluated to develop a diagnosis of specific disease and grade. Orthopaedic physical examination is also performed for evaluation of degree of recovery after treatment. Table 1 shows a list of representative items in orthopaedic physical examination for the evaluation of the knee joint. There are proper orthopaedic physical examination methods for each of the 4 major ligaments (ACL, PCL, MCL, LCL). Orthopaedic physical examination plays an important role in diagnosis, but a number of items in orthopaedic physical examination depend on qualitative judgment. Thus in addition to the problems above mentioned, there is an issue of difficulty in educating younger physicians on techniques of proficient physicians with experience in orthopaedic physical examination. In such a situation, the need to develop quantitative evaluation for orthopaedic physical examination has increased.

Table 1 Typical orthopaedic physical examinations for knee ligament injury

\begin{tabular}{c|c}
\hline Ligament & Type of examination \\
\hline Anterior cruciate ligament (ACL) & Anterior drawer test, Lachman test \\
\hline Posterior cruciate ligament (PCL) & Posterior drawer test, Reverse Lachman test \\
\hline Medial collateral ligament (MCL) & Valgus stress test \\
\hline Lateral collateral ligament (LCL) & Varus stress test \\
\hline
\end{tabular}

\subsection{Existing researches on quantification of orthopaedic physical examination}

$\mathrm{X}$-ray stress test is employed to quantitatively evaluate orthopaedic physical examination $^{(2)}$. This method employs $\mathrm{X}$-ray examination and sometimes special joint fixture to measure the displacement or angle of the tibia for diagnosis ${ }^{(3)}$. However, this method has the disadvantage of increased examination time, patient exposure, and reduced reproducibility due to differences in the incident angle of the x-ray. The KT-1000 Arthrometer ${ }^{(4),(5)}$ from MEDmetric allows examination without exposure. This device is used for quantitatively evaluating the Lachman test and anterior drawer test. It applies a certain anterior drawer force to the tibia and confirms anterior displacement. The generic KT-2000 Arthrometer is extensively used in clinical settings ${ }^{(6)}$; however the type of examination possible using such a special device is limited. For example, the KT-2000 Arthrometer can be used only for examination of ACL injury and not for other ligament injury.

\subsection{Concept of the system}

The characteristics of the orthopaedic physical examination assisting system to be developed in this study lie in the quantification of examination behavior itself without going 
over for examination method so far. Fig. 1 shows the image of the system we propose. It is a system that measures force vectors applied by the physician and motion of the knee joint using three-dimensional position measurement equipment to indicate the information on a monitoring screen in real time, and to record in orthopaedic physical examination to diagnose joint laxity of the knee. By feeding back the position and magnitude of force vectors input by the physician and posture of the subject knee to the physician, improvement of accuracy and reproducibility of examination is expected. Quantification does not require a change to the conventional examination method which has already spread world-wide, and this corresponds to multiple examinations. Therefore, this concept is considered appropriate for the current clinic-al state.

Force vectors are measured by a special device attached to the hand of the physician. A large scale device prevents natural orthopaedic physical examination as usual and further tends to elongate the examination time. Therefore, it is necessary to use a device easily worn, not hindering the senses of the hand. Since the knee joint has degrees of freedom not only for flexion/extension but also for varus/valgus, internal/external rotations, furthermore anterior/posterior, lateral/medial, and in the direction of the long axis, which are relative displacements between the femur and tibia, it is necessary to measure 6 DOF motion (motion with 6 degrees of freedom) less invasively in order to quantify the motion of the knee joint.

This system also can be used as a training tool for younger physicians by measuring the orthopaedic physical examination conducted by the skillful practitioners for use as teaching data. As the related study, there is a lower extremity robot for rehabilitation practice ${ }^{(7)}$ by Makita et al. Kikuchi et al. have also developed a $\operatorname{robot}^{(8)}$ to reproduce the spasmodic leg by using an MR clutch. Hayashi et al. developed a lower extremity $\operatorname{robot}^{(9)}$ which reproduces flexion/extension and internal/external rotation motions of the knee joint by utilizing the leg of a robot walking with two legs, aiming at training physical/ occupational therapists. Vankipuram et al. developed a virtual reality orthopaedic drilling simulator ${ }^{(10)}$ that is designed to provide visiohaptic interaction with virtual bones. Frey et al. developed a virtual reality knee simulator $^{(11),(12)}$ using haptic technology. However, these systems reproduce the virtual human leg or virtual 6 DOF motion of the joint. Our system is able to quantitates real human joint motion, this point has great advantage for younger physicians to realize practical training.

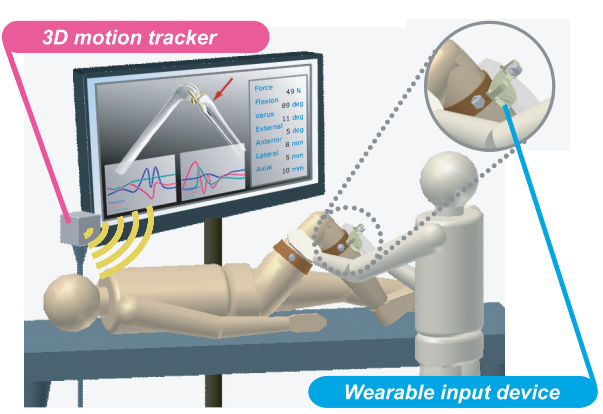

Fig. 1 Orthopaedic physical examination assisting system

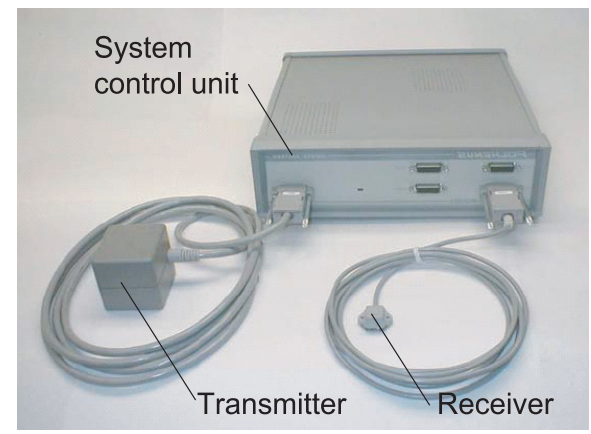

Fig. 2 6DOF magnetic tracker FASTRAK (Polhemus, USA)

\section{Outline of the orthopaedic physical examination assisting system}

\subsection{Components of the system}

The developed system is composed of 4 elements.

( 1 ) Three-dimensional position measuring equipment: To measure 6 DOF knee motion and the position/posture of the device described below.

( 2 ) Wearable input device: Worn on the examiner's hand to measure force vectors input against the knee. 
( 3 ) Feature point acquisition stylus: To measure feature point coordinates to be used for registration of the knee joint coordinate system.

( 4 ) PC/monitor: Various computations including coordinate transformation and indication/record of data.

Each element and measuring method of 6 DOF knee motion and information shown on the monitor screen are described below.

\subsection{Selection of three-dimensional position measuring equipment}

Specifications for the equipment include the following items.

(1) It is possible to measure 6 DOF knee motion.

(2) It is not easily affected by the shields of equipments in the room or operation room or patients or physicians themselves.

Based on the above, we determined to use FASTRAK (Fig. 2), Magnetic three-dimensional position measuring equipment from POLHEMUS, in this study. FASTRAK receives the alternative magnetic field emitted from a transmitter through the receiver, and measures the three-dimensional position of the receiver and Euler angle. It requires smaller space for installation, thus can measure without being influenced by physical obstacles. Measurement precision (RMS) is $0.76 \mathrm{~mm}$ for position and 0.15 degrees for angle. The obtained Euler angle (Azimuth, Elevation, Roll) is determined in Z-Y-X axis system.

\subsection{Wearable input device}
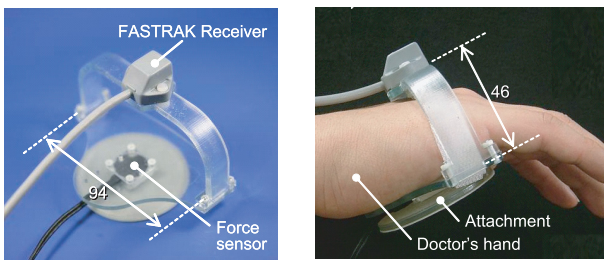

Fig. 3 Wearable input device with FASTRAK receiver and force sensor
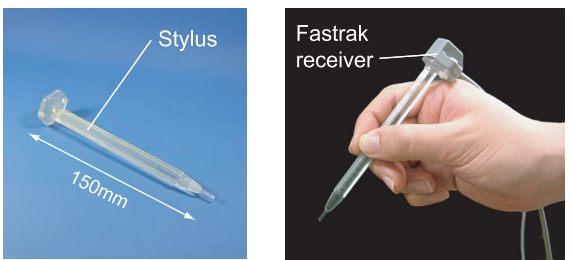

Fig. 4 Feature point acquisition stylus with FASTRAK receiver

Fig. 3 shows the wearable input device we have developed. It is composed of a force sensor and FASTRAK receiver in order to measure force vectors produced on the palm of the physician. It is a compact and thin design to allow natural orthopaedic physical examination. The force sensor used is a small 3-axis force sensor 5mm in thickness (USL-H5-200N: Tec Gihan) which can detect vertical and shearing force. The force applied by physicians in examination varies, and this sensor, with a $200 \mathrm{~N}$ rating in the vertical direction, is considered to sufficiently meet the specification, considering that the KT-2000 Arthrometer can apply a maximum load of approximately $140 \mathrm{~N}$. In this device, a force sensor and a receiver are set out of the range influenced by the magnetic field of FASTRAK (40mm or higher: measured experimentally). The material of the device is resin (produced by rapid prototyping).

\subsection{Feature point acquisition stylus}

In order to define the joint coordinate system, it is necessary to acquire the feature point coordinates of the lower body. Fig. 4 shows the feature point acquisition device. Taking into consideration the influence on the magnetic field, it is made of resin and the receiver is fixed with resin nuts and screws.

\subsection{PC/monitoring device}

Fig. 5 shows the entire system composed of PC and devices. The PC is a notebook type with all-in-one monitor. The measuring cycle of FASTRAK is $30 \mathrm{~Hz}$. Developing language is $\mathrm{C}++$. 


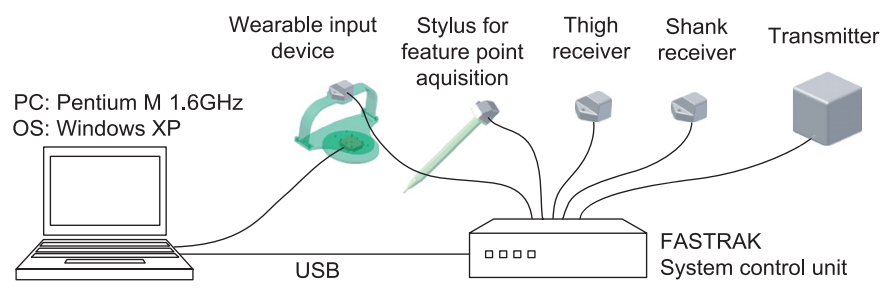

Fig. 5 System diagram of Orthopaedic physical examination assisting system

\subsection{Definition of knee joint coordinate system}

The coordinate system most generally used in orthopaedics is the Grood coordinate system ${ }^{(13)}$; however, it has a problem in using points which cannot be percutaneously obtained, such as in the femoral head, for definition of the coordinate system. Therefore, we adopt the coordinate system ${ }^{(14)}$ defined by easily palpable anatomical feature points proposed by Hoshino et al. Feature points (Fig. 6) include the following 7 points; Point A: Greater trochanter, Point B: Medial epicondyle, Point C: Lateral epicondyle, Point D: Intersection of medial collateral ligament (MCL) and knee joint line, Point E: Proximal end of fibula, Point F: Medial malleolus, and Point G: Lateral malleolus. The coordinate system is defined as follows:

( 1 ) Femur coordinate system $\Sigma_{F e m u r}$

- Original point: Middle point between Point B and Point C.

- Z-axis: An axis connecting original point and Point A.

- X-axis: An axis existing on the plane passing the original point, Point $\mathrm{B}$, and Point $\mathrm{C}$ (femoral frontal plane), and vertical to $\mathrm{z}$-axis (right side is front side regardless of right or left).

- Y-axis: An axis produced by the vector product of $\mathrm{z}$-axis and $\mathrm{x}$-axis.

( 2 ) Tibia coordinate system $\Sigma_{\text {Tibia }}$

- Original point: Middle point between Point D and Point E.

- $\mathrm{z}$-axis: An axis connecting the original point and the intersection of Point $\mathrm{F}$ and Point G.

- $x$-axis: An axis existing on the plane passing the original point, Point $\mathrm{D}$, and Point $\mathrm{E}$ (tibial frontal plane), and vertical to $\mathrm{z}$-axis (the right side is the front side regardless of right or left).

- $y$-axis: An axis produced by the vector product of $\mathrm{z}$-axis and $\mathrm{x}$-axis.

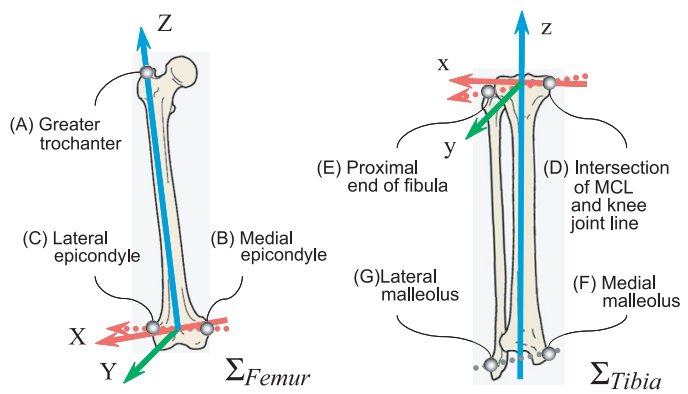

Fig. 6 Femoral and tibial Cartesian coordinate system with bony landmarks

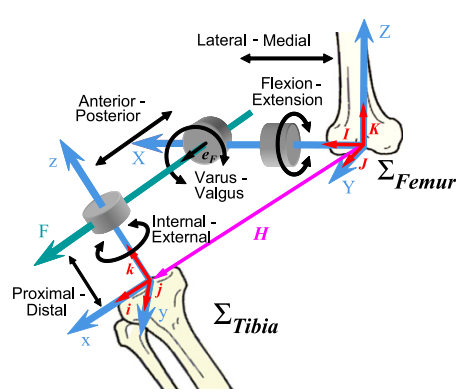

Fig. 7 Knee joint angles and kinematics proposed by Grood

Furthermore, Fig. 7 shows the model of 6 DOF knee motion which has a floating axis F making a right angle with both the x-axis of $\Sigma_{F e m u r}$ and the $\mathrm{z}$-axis of $\Sigma_{\text {Tibia }}$. 6 DOF knee motion is defined as follows: where let the vector connecting the original point of $\Sigma_{F e m u r}$ and the original point of $\Sigma_{\text {Tibia }}$ be $\boldsymbol{H}$, the base vector of $\Sigma_{\text {Femur }}$ be $(\boldsymbol{i}, \boldsymbol{j}, \boldsymbol{k})$, the base vector of $\Sigma_{\text {Tibia }}$ 
be $(\boldsymbol{i}, \boldsymbol{j}, \boldsymbol{k})$, and the base vector of floating axis F be $\boldsymbol{e}_{F}=(\boldsymbol{k} \times \boldsymbol{I}) /\|\boldsymbol{k} \times \boldsymbol{I}\|$.

$$
\begin{aligned}
& \alpha=-\sin ^{-1}\left(\boldsymbol{K} \cdot \boldsymbol{e}_{F}\right) \\
& \beta=\cos ^{-1}(\boldsymbol{I} \cdot \boldsymbol{k})-\pi / 2 \\
& \gamma=-\sin ^{-1}\left(\boldsymbol{i} \cdot \boldsymbol{e}_{F}\right) \\
& q_{1}=\boldsymbol{H} \cdot \boldsymbol{I} \\
& q_{2}=\boldsymbol{H} \cdot \boldsymbol{e}_{F} \\
& q_{3}=-\boldsymbol{H} \cdot \boldsymbol{k}
\end{aligned}
$$

Where, $\alpha$ : flexion angle, $\beta$ : varus angle (in the case of the right knee), $\gamma$ : external rotation angle (in the case of the right knee), $q_{1}$ : lateral displacement magnitude, $q_{2}$ : anterior displacement magnitude, and $q_{3}$ : distal displacement magnitude.

\subsection{Coordinate transformation of the device stylus}

In order to obtain force vector $\left({ }^{T r} \boldsymbol{f}\right)$ of the wearable input device and the position vector $\left({ }^{T r} \boldsymbol{d}\right)$ of its action point in a transmitter coordinate system $\Sigma_{\text {Trans }}$, and the tip position vector $\left({ }^{T} \boldsymbol{S}\right)$ of a feature point acquisition stylus, the coordinate transformation in Fig.8 is required. Using the rotation matrix around each axis, $\boldsymbol{R}$, and the parallel translation matrix $\boldsymbol{T}_{0}$

$$
\boldsymbol{T}_{0}=\left(\begin{array}{llll}
1 & 0 & 0 & x \\
0 & 1 & 0 & y \\
0 & 0 & 1 & z \\
0 & 0 & 0 & 1
\end{array}\right)
$$

from the three-dimensional position $(x, y, z)$ and the Euler angle (Azimuth, Elevation, Roll) (hereinafter abbreviated as $(A, E, R)$ ) which are obtained from the FASTRAK receiver, the rotation matrix $\left({ }^{T r} \boldsymbol{R}_{R e}\right)$ and the homogeneous transformation matrix $\left({ }^{T r} \boldsymbol{T}_{R e}\right)$ from the receiver coordinate system to $\Sigma_{\text {Trans }}$ are expressed by the following equation:

$$
\begin{aligned}
{ }^{T r} \boldsymbol{R}_{R e} & =\boldsymbol{R}_{z}^{-1}(A) \boldsymbol{R}_{y}^{-1}(E) \boldsymbol{R}_{x}^{-1}(R) \\
{ }^{T r} \boldsymbol{T}_{R e} & =\boldsymbol{T}_{0} \boldsymbol{R}_{z}^{-1}(A) \boldsymbol{R}_{y}^{-1}(E) \boldsymbol{R}_{x}^{-1}(R)
\end{aligned}
$$

Using equations (8) and (9), it is possible to calculate ${ }^{T r} \boldsymbol{R}_{D e},{ }^{T r} \boldsymbol{R}_{S t},{ }^{T r} \boldsymbol{T}_{D e}$, and ${ }^{T r} \boldsymbol{T}_{S t}$. Further ${ }^{D e} \boldsymbol{T}_{F o},{ }^{D e} \boldsymbol{d}$, and ${ }^{S t_{\boldsymbol{S}}}$ are well known in the designing process; and force vector ${ }^{F o} \boldsymbol{f}$ in force sensor coordinate system $\Sigma_{\text {Force }}$ is directly obtained, thus ${ }^{T r} \boldsymbol{f},{ }^{T r} \boldsymbol{d}$, and ${ }^{T r_{\boldsymbol{S}}}$ are obtained from the following equations:

$$
\begin{aligned}
& { }^{T r} \boldsymbol{f}={ }^{T r} \boldsymbol{R}_{D e}{ }^{D e} \boldsymbol{R}_{F o}{ }^{F o} \boldsymbol{f} \\
& { }^{T r} \boldsymbol{d}={ }^{T r} \boldsymbol{T}_{D e}{ }^{D e} \boldsymbol{d} \\
& T r_{\boldsymbol{S}}={ }^{T r} \boldsymbol{T}_{S t}{ }^{S t} \boldsymbol{s}
\end{aligned}
$$

\subsection{Registration of the coordinate system and measurement of knee motion}

As shown in Fig. 9, fix the receivers on the femoral part and lower leg part with the velcro fastener. Assuming the relative position between the femur and femoral receiver, and that between the tibia and lower leg receiver are constant, it is possible to calculate the position/posture of $\Sigma_{\text {Femur }}$ and $\Sigma_{\text {Tibia }}$ during motion from each receiver's data by obtaining the homogeneous transformation matrix between $\Sigma_{F e m u r}$ and the femoral receiver coordinate system $\Sigma_{\text {Thigh }},{ }^{T h} \boldsymbol{T}_{F e}$, and the homogeneous transformation matrix between $\Sigma_{\text {Tibia }}$ and the lower 


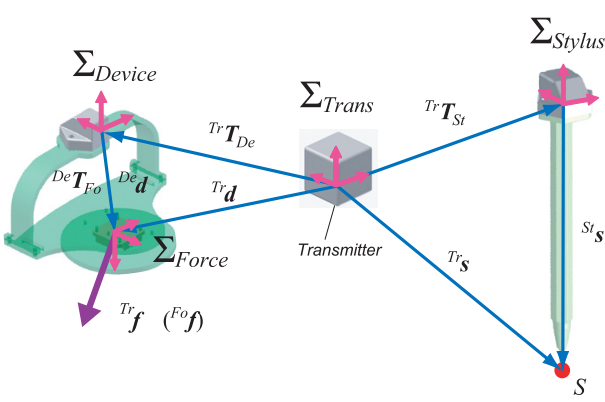

Fig. 8 Coordinate transformation of the wearable input device and the feature point acquisition stylus

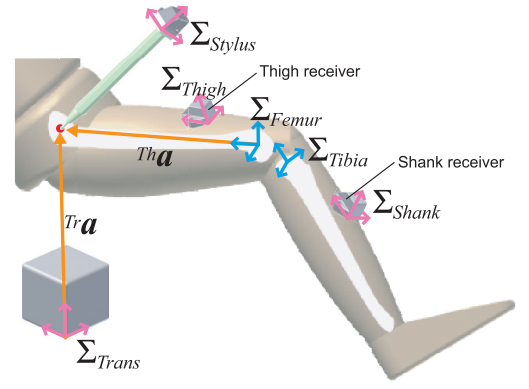

Fig. 9 Registration of the femoral and tibial coordinate system with FASTRAK

leg receiver coordinate system $\Sigma_{S h a n k},{ }^{S} \boldsymbol{T}_{T i}$, and by registering the data. Firstly, measure feature point coordinates in the $\Sigma_{\text {Thigh }}$ and $\Sigma_{\text {Shank }}$ systems (hereinafter referred to as sampling). We will explain using the greater trochanter (Point A), one of the sampling points, as an example. The position vector of Point A in $\Sigma_{\text {Trans }},{ }^{T r} \boldsymbol{a}$, the position vector of Point A in $\Sigma_{\text {Thigh }},{ }^{T h} \boldsymbol{a}$, and, the homogeneous transformation matrix from $\Sigma_{\text {Thigh }}$ to $\Sigma_{\text {Trans }},{ }^{T} \boldsymbol{T}_{T h}$ meet the following equation:

$$
{ }^{T r} \boldsymbol{a}={ }^{T r} \boldsymbol{T}_{T h}{ }^{T h} \boldsymbol{a}
$$

Since ${ }^{T r} \boldsymbol{a}$ is obtained from equation (12), and ${ }^{T} \boldsymbol{T}_{T h}$ is obtained from femoral receiver data and equation (9), ${ }^{T h} \boldsymbol{a}$ is obtained from the following equation:

$$
{ }^{T h} \boldsymbol{a}={ }^{T r} \boldsymbol{T}_{T h}^{-1 T r} \boldsymbol{a}
$$

Similarly, the position vectors of Points B and C in $\Sigma_{T h i g h},{ }^{T h} \boldsymbol{b},{ }^{T h} \boldsymbol{c}$, and the position vectors of Points D to G in $\Sigma_{S h a n k},{ }^{S h} \boldsymbol{d}$ to ${ }^{S h} \boldsymbol{g}$, respectively, are calculated. From the definition of the coordinate system described in Section 3.6 and the position vectors above mentioned, ${ }^{T h} \boldsymbol{T}_{F e}$ and ${ }^{S h} \boldsymbol{T}_{T i}$, are obtained as follows.

( 1 ) Femur coordinate system $\Sigma_{\text {Femur }}$

Let the original point and base vector of $\Sigma_{\text {Femur }}$ in the $\Sigma_{\text {Thigh }}$ system, and the base vector of the axis connecting Point B and Point $\mathrm{C}$ be ${ }^{T h} \boldsymbol{o},\left({ }^{T h} \boldsymbol{I},{ }^{T h} \boldsymbol{J},{ }^{T h} \boldsymbol{K}\right)$, and ${ }^{T h} \boldsymbol{I}^{\prime}$, respectively, ${ }^{T h} \boldsymbol{o}$ is expressed as follows:

$$
\begin{aligned}
& { }^{T h} \boldsymbol{O}=\left({ }^{T h} \boldsymbol{b}+{ }^{T h} \boldsymbol{c}\right) / 2 \\
& { }^{T h} \boldsymbol{K}=\left({ }^{T h} \boldsymbol{a}-{ }^{T h} \boldsymbol{o}\right) /\left\|^{T h} \boldsymbol{a}-{ }^{T h} \boldsymbol{o}\right\| \\
& { }^{T h} \boldsymbol{I}^{\prime}=\left({ }^{T h} \boldsymbol{c}-{ }^{T h} \boldsymbol{b}\right) /\left\|^{T h} \boldsymbol{c}-{ }^{T h} \boldsymbol{b}\right\| \\
& { }^{T h} \boldsymbol{J}=\left({ }^{T h} \boldsymbol{K} \times{ }^{T h} \boldsymbol{I}^{\prime}\right) /\left\|^{T h} \boldsymbol{K} \times{ }^{T h} \boldsymbol{I}^{\prime}\right\| \\
& { }^{T h} \boldsymbol{I}={ }^{T h} \boldsymbol{J} \times{ }^{T h} \boldsymbol{K}
\end{aligned}
$$

Therefore, ${ }^{T h} \boldsymbol{T}_{F e}$ becomes the following equation:

$$
{ }^{T h} \boldsymbol{T}_{F e}=\left(\begin{array}{ccc|c}
{ }^{T h} \boldsymbol{I} & { }^{T h} \boldsymbol{J} & { }^{T h} \boldsymbol{K} & { }^{T h} \boldsymbol{o} \\
\hline 0 & & 1
\end{array}\right)
$$

( 2 ) Tibia coordinate system $\Sigma_{\text {Tibia }}$

Let the original point and base vector of $\Sigma_{\text {Tibia }}$ in the $\Sigma_{\text {Shank }}$ system, and the base vector of axis connecting Point D and Point E be ${ }^{S h} \boldsymbol{o},\left(S h_{\boldsymbol{i}},{ }^{S h} \boldsymbol{j},{ }^{S h} \boldsymbol{k}\right)$, and ${ }^{S \boldsymbol{i}^{\prime}}$, respectively, ${ }^{S h} \boldsymbol{o}$ is expressed as follows:

$$
\begin{aligned}
S h_{\boldsymbol{o}} & =\left({ }^{S h} \boldsymbol{d}+{ }^{S h} \boldsymbol{e}\right) / 2 \\
S h_{\boldsymbol{k}} & =\left\{{ }^{S h} \boldsymbol{o}-\left({ }^{S h} \boldsymbol{f}+{ }^{S h} \boldsymbol{g}\right) / 2\right\} /\left\|{ }^{S h} \boldsymbol{o}-\left({ }^{S h} \boldsymbol{f}+{ }^{S h} \boldsymbol{g}\right) / 2\right\| \\
S h_{\boldsymbol{i}} & =\left({ }^{S h} \boldsymbol{e}-{ }^{S h} \boldsymbol{d}\right) /\left\|{ }^{S h} \boldsymbol{e}-{ }^{S h} \boldsymbol{d}\right\|
\end{aligned}
$$




$$
\begin{aligned}
& S h \boldsymbol{j}=\left({ }^{S h} \boldsymbol{k} \times S h_{\boldsymbol{i}}\right) /\left\|^{S h} \boldsymbol{k} \times S \boldsymbol{i}^{\prime}\right\| \\
& S \boldsymbol{i}_{\boldsymbol{i}}=S{ }_{\boldsymbol{j}} \times{ }^{S h} \boldsymbol{k}
\end{aligned}
$$

Therefore, ${ }^{S h} \boldsymbol{T}_{T i}$ becomes the following equation:

$$
{ }^{S h} \boldsymbol{T}_{T i}=\left(\begin{array}{ccc|c}
S h_{\boldsymbol{i}} & S{ }^{\boldsymbol{j}} & S h_{\boldsymbol{k}} & S h_{\boldsymbol{o}} \\
\hline & 0 & & 1
\end{array}\right)
$$

When ${ }^{T} \boldsymbol{T}_{F e}$ is placed as a homogeneous transformation matrix from $\Sigma_{F e m u r}$ to $\Sigma_{\text {Trans }}$, and ${ }^{T}{ }^{T} \boldsymbol{T}_{T i}$ is placed as a homogeneous transformation matrix from $\Sigma_{\text {Tibia }}$ to $\Sigma_{T r a n s}$, The following equations are established:

$$
\begin{aligned}
& { }^{T r} \boldsymbol{T}_{F e}={ }^{T r} \boldsymbol{T}_{T h}{ }^{T h} \boldsymbol{T}_{F e} \\
& { }^{T r} \boldsymbol{T}_{T i}={ }^{T r} \boldsymbol{T}_{S h}{ }^{S h} \boldsymbol{T}_{T i}
\end{aligned}
$$

${ }^{T}{ }^{T} \boldsymbol{T}_{S h}$ is obtained from the lower leg receiver and equation (9) similarly to ${ }^{T} \boldsymbol{T}_{T h} .6$ DOF knee motion can be obtained by transforming $\left({ }^{T h} \boldsymbol{I},{ }^{T h} \boldsymbol{J},{ }^{T h} \boldsymbol{K}\right)$ and $\left({ }^{S} \boldsymbol{i}_{\boldsymbol{i}},{ }^{S h} \boldsymbol{j},{ }^{S h} \boldsymbol{k}\right)$ to the $\Sigma_{\text {Trans }}$ system using ${ }^{T} \boldsymbol{T}_{F e}$ and ${ }^{T} \boldsymbol{T}_{T i}$, respectively and by substituting them to equations (1) to (6).

\subsection{Information shown by system}

The developed system shows the following two sets of information in real-time:

( 1 ) 6 DOF knee motion: By showing 6 DOF motion obtained from equations (1) to (6), it is possible to perform examination, grasping the results. It is reported that in joint laxity examination, even if the outer force applied to the tibia is constant, distribution of soft tissue tensile in the inside of the joint changes depending on the joint posture such as flexion angle or inter$\mathrm{nal} /$ external rotation angles ${ }^{(15)-(17)}$, thus this is also essential information in the performance of examination with reproducibility.

( 2 ) Force vector added to the tibia: By showing the magnitude of force, it is possible for examiners to adjust the loading force, which enables the examination with high accuracy and reproducibility. Generally, in orthopaedic physical examination of the knee, force is applied to the tibia, the action point of force is shown after transformation into the tibia coordinate system using the following equation:

$$
{ }^{T i} \boldsymbol{d}={ }^{T i} \boldsymbol{T}_{T r}{ }^{T i} \boldsymbol{T}_{D e}{ }^{D e} \boldsymbol{d}
$$

${ }^{T} \boldsymbol{T}_{T r}$ in the above equation is the reverse matrix of ${ }^{T} \boldsymbol{T}_{T i}$ which was obtained from equation (20).

These pieces of information are shown on the console screen of the PC as Fig. 10.

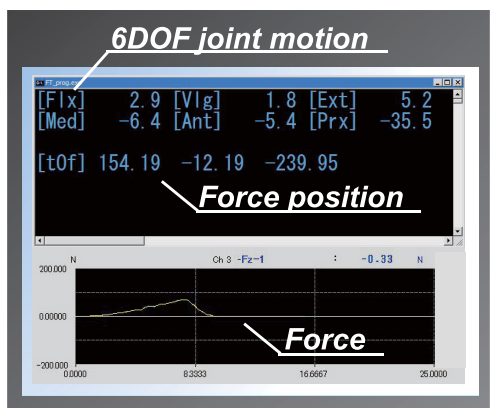

Fig. 10 Console information acquired by system. 6DOF joint motion, force position in the tibial coordinate system and force value. 
4. Experiments to evaluate effectiveness of orthopaedic physical examination assisting system.

\subsection{Purpose of experiments}

The purpose of this experiment with joint laxity examination as a target is to evaluate the accuracy and reproducibility of examination in cases without information shown by orthopaedic physical examination assisting system and in cases with referencing information, and to demonstrate the effectiveness of this system in the evaluation of joint laxity. The experiment also aims at evaluating for training effect of this system.

\subsection{Subject examination}

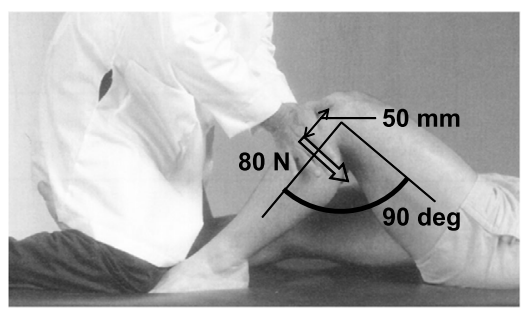

Fig. 11 Experimental condition of posterior drawer test

This experiment examined the posterior drawer test (Fig. 11), one of joint laxity examinations. Posterior drawer test is used in orthopaedic physical examination to evaluate joint laxity caused by posterior cruciate ligament injury. The general posterior drawer test is conducted as follows:

( 1 ) Confirm that the patient's hamstring is sufficiently relaxed with the knee joint flexed at 90 degrees.

( 2 ) Add posterior drawer force to the upper end of the tibia (50mm distally from the knee joint line) $(80 \mathrm{~N})$

( 3 ) The healthy knee should also be examined similarly.

If the drawer force of the injured knee is $5 \mathrm{~mm}$ or longer than that of the healthy knee, posterior cruciate ligament injury is diagnosed. Posterior drawer force of the tibia and its loading position are based on proceeding studies and literature values ${ }^{(18)-(20)}$.

\subsection{Experimental method}

Five young, healthy individuals each were selected as subjects playing the role of patient and examiner. Prior to participation in the experiment, the content was clearly explained and consent was obtained. In order to minimize FASTRAK measurement error caused by magnet field interference, a wooden examination table was used, and the system was set away from the wall and floor, which has reinforced steel, and from metallic desks. The experiment was performed in accordance with the following procedure:

(1) The receiver was attached to the femur and lower leg of subjects playing the role of patient. Sampling with the feature point acquisition stylus was performed, and the coordinates were registered.

( 2 ) Subjects playing the role of patient were directed to lie in a supine position with natural posture. This state is defined as the initial state (Fig. 12(A)). Subjects playing the role of patient were directed to relax their muscles and return to the initial condition once the examination was completed.

( 3 ) Subjects playing the role of examiner attached the wearable input device and performed the posterior drawer test. The test was performed a total of 6 times in 3 sets of 2 measurements each (Fig. 12(B))

- First set: The examination was performed without using information obtained by orthopaedic physical examination assisting system. 
- Second set: Examination confirming information obtained by orthopaedic physical examination assisting system was performed.

- Third set: Training on posterior drawer examination was conducted for several minutes, confirming information obtained by orthopaedic physical examination assisting system, then examination was performed similarly to the first set without using information from the system.

As the measurement data, knee flexion angle and load position when loading force becomes maximum during examination were extracted. With regard to the accuracy of examination, absolute errors from the target values of knee flexion angle (90deg), loading force $(80 \mathrm{~N})$, and loading position $(50 \mathrm{~mm})$ were selected as indices. With regard to the reproducibility of examination, differences in each item of one set on the same examiner were selected as indices.
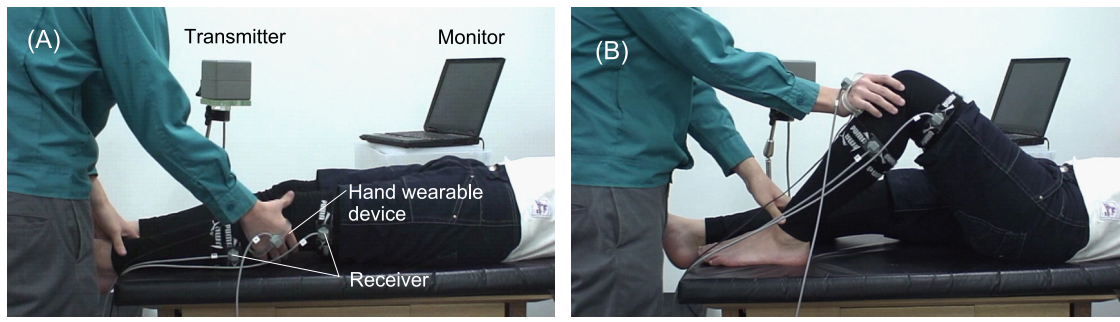

Fig. 12 Overview of knee laxity diagnosis by orthopaedic physical examination assisting system. (A): Initial position. (B): Examination position.

\subsection{Experiment results}

4.4.1. Comparison between the presence and absence of orthopaedic physical examination assisting system

(1) Accuracy: Fig. 13 shows a comparison of absolute errors from target values for each item between the presence and absence of orthopaedic physical examination assisting system. In case of presence of the system, mean error values from the target values were significantly decreased in all items of knee flexion angle, loading force, and loading position when compared with absence of the system. In particular, significant results was obtained in the loading position. The ratio (relative error) of mean error value against the target value was decreased, respectively, from $9.4 \%(8.5 \mathrm{deg})$ to $1.5 \%(1.3 \mathrm{deg})$ in knee flexion angle, $31.4 \%$ $(25.1 \mathrm{~N})$ to $6.4 \%(5.1 \mathrm{~N})$ in loading force, and $78.9 \%(39.5 \mathrm{~mm})$ to $1.4 \%(0.7 \mathrm{~mm})$ in loading position.

(2) Reproducibility: Fig. 14 shows the absolute errors between the first and second examinations in each item in the same examiner in the presence and absence of orthopaedic physical examination assisting system. In case of presence of system, mean error value between first and second examinations was significantly decreased in knee flexion angle, particularly significantly decreased in loading force, while not significantly decreased in loading position when compared with that for absence of the system. Mean error value was decreased, respectively, from $2.2 \mathrm{deg}$ to $0.5 \mathrm{deg}$ in knee flexion angle, from $30.3 \mathrm{~N}$ to $5.9 \mathrm{~N}$ in loading force, and from $3.6 \mathrm{~mm}$ to $0.8 \mathrm{~mm}$ in loading position.

\subsubsection{Comparison between before and after training}

(1) Accuracy: Fig.15 shows comparison of absolute errors from the target values of each item between before and after training using orthopaedic physical examination assisting system. After training, mean error value from target value was significantly decreased in knee flexion angle and, in particular, significantly decreased in position error. No significant decrease was observed in loading position. The ratio (relative error) of mean error value against target value was decreased, respectively, from $9.4 \%$ (8.5deg) to $3.5 \%$ (3.1 eg) in knee flexion angle, and from $78.9 \%(12.1 \mathrm{~mm})$ to $9.4 \%(4.7 \mathrm{~mm})$ in loading position. However, loading force was increased from $31.4 \%(25.1 \mathrm{~N})$ to $60.0 \%(47.8 \mathrm{~N})$. 

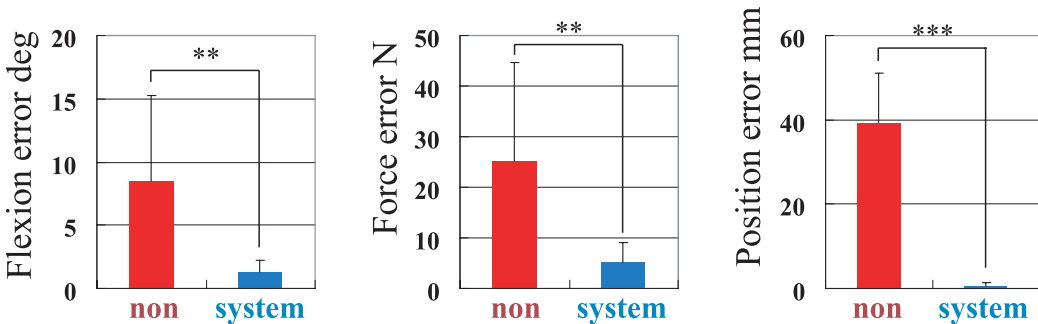

Fig. 13 Comparison of difference from indicated value with or without the system. $* * \mathrm{P}<0.01, * * * \mathrm{P}<0.001, \mathrm{n}=10, \mathrm{t}-$ Student test.
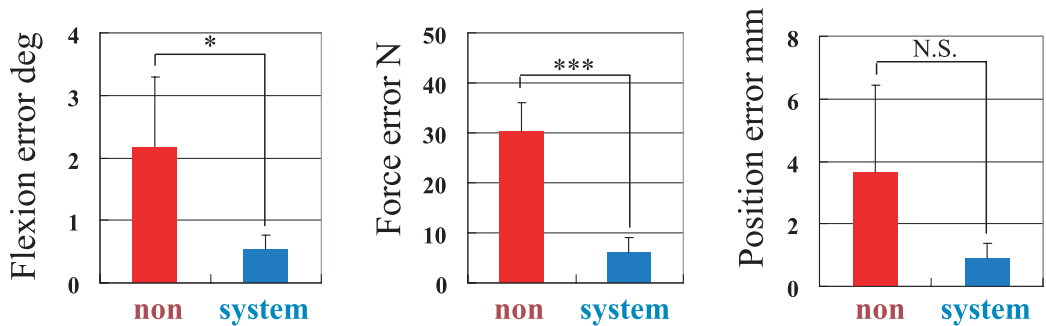

Fig. 14 Comparison of reproducibility error with or without the system. $* \mathrm{P}<0.05$, $* * * \mathrm{P}<0.001, \mathrm{n}=5, \mathrm{t}$-Student test.

( 2 ) Reproducibility: Fig.16 shows the absolute errors between the first and second examinations in each item in the same examiner before and after training using orthopaedic physical examination assisting system. After training, mean error value was significantly decreased between the first and second examinations in loading force, while not significantly decreased in knee flexion angle and loading position when compared with before training. Mean error value was decreased from $2.2 \mathrm{deg}$ to $1.2 \mathrm{deg}$ in flexion degree, and from $30.3 \mathrm{~N}$ to $15.1 \mathrm{~N}$ in loading force, while being slightly increased from $3.6 \mathrm{~mm}$ to $3.8 \mathrm{~mm}$ in loading position.
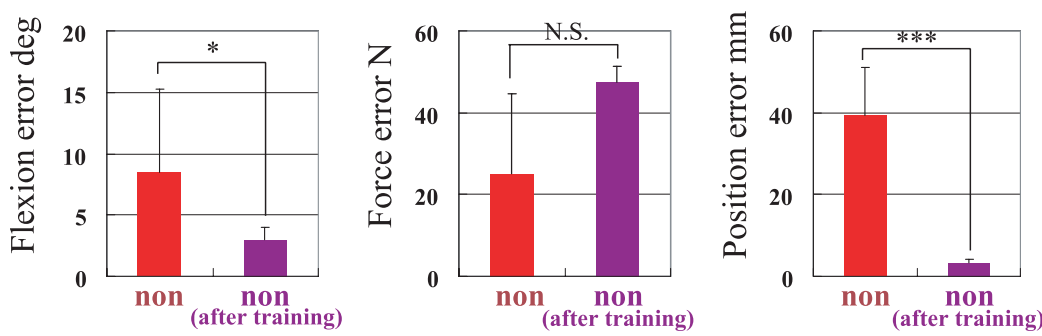

Fig. 15 Comparison of difference from indicated value before and after training with the system. ${ }^{*} \mathrm{P}<0.05, * * * \mathrm{P}<0.001, \mathrm{n}=10$, t-Student test.

\subsection{Discussion}

The results in Fig. 13 clarified that use of orthopaedic physical examination assisting system significantly improved accuracy in all items such as knee flexion angle, loading force, and loading position. The results in Fig. 14 clarified that reproducibility was significantly improved in knee flexion angle, and loading force. Furthermore, the results in Fig. 15 revealed that training using orthopaedic physical examination assisting system significantly improved accuracy in knee flexion angle, and loading position. The following is a discussion of the results obtained from this study by comparing with Weber ratio. Existing studies have attempted to clarify the corresponding relation between stimulation from external and internal senses. When basic stimulation is indicated by $I$, and discrimination threshold (amount suffi- 

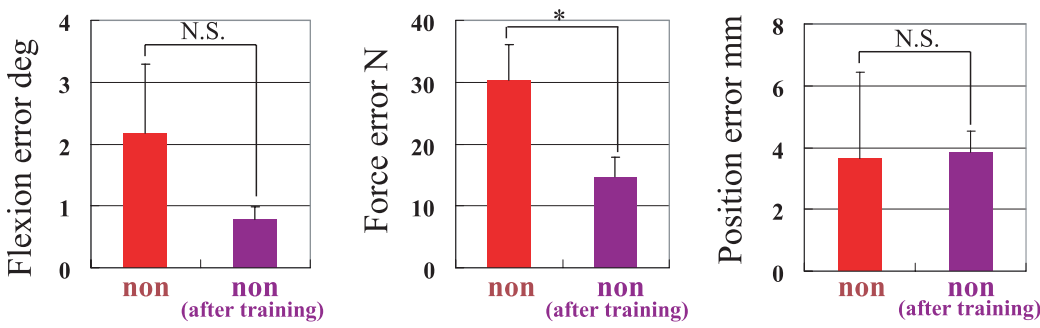

Fig. 16 Comparison of reproducibility error before and after training with the system. $* \mathrm{P}<0.05, \mathrm{n}=5, \mathrm{t}-$ Student test.

cient to enable discrimination) by $\Delta I$, the following equation is established:

$$
K=\Delta I / I
$$

The constant value $K$ is called the Weber ratio. According to existing studies ${ }^{(21)}$, the Weber ratio for strength of pressure is 0.143 , and the Weber ratio for discrimination of length of line segment is 0.029 , showing that visual sense can discriminate the object approximately 5 times more accurately than strength sense. Calculation of the Weber ratio regarding results of Fig. 13 revealed a loading force of $0.06(\Delta I: 5.1 \mathrm{~N}, I: 80 \mathrm{~N})$ and a loading position of 0.01 $(\Delta I: 0.7 \mathrm{~mm}, I: 50 \mathrm{~mm})$. These values are better Weber ratios than those shown in the literature ${ }^{(21)}$, indicating that use of this system enables orthopaedic physical examination with a degree of accuracy that exceeds general human capacity. The result of reproducibility in loading position is not significantly improved. It is thought that reproducibility using the visual sense may be relatively high from the beginning. Calculation of Weber ratio for the results of Fig. 15 revealed a loading force of $0.60(\Delta I: 47.8 \mathrm{~N}, I: 80 \mathrm{~N})$ and a loading position of $0.09(\Delta I: 4.7 \mathrm{~mm}$, $I: 50 \mathrm{~mm}$ ). The learning effect of items which can be visually discriminated, such as knee flexion angel and loading position, is higher than that of loading force which is discriminated by force sense, which may be correlated with Weber ratio. Intuitively, with regard to knee flexion angel and loading position, the situation at training tends to be memorized easily from the surrounding background or landmark of the lower leg, while with regard to loading force, it has to depend on purely memorized sense alone because there is nothing to be simultaneously compared, which may increase error from the target value. In this experiment, training time was only several minutes, and we would like to examine the learning effect of force sense in long term training in the future.

\section{Summary}

In this study, we developed an orthopaedic physical examination assisting system aiming at improvement in the accuracy and reproducibility of joint laxity diagnosis. We performed experiments to evaluate the posterior drawer test and confirmed that use of the system enables examination on knee flexion angle, loading force and position on the tibia with high accuracy and reproducibility. We also evaluated learning effect by training, which revealed that accuracy is increased in knee flexion angle and loading position.

The perspective of this study includes the following:

(1) Evaluation in clinical setting: To develop quantitative diagnosis criteria by clinically using this system and performing examination on actual patients.

( 2 ) Application to other examinations: In this study, only posterior drawer test was covered. Hereafter, we will aim at extending the scope of study to examinations on other knee joint laxity diagnosis or examination of other sites and establishing this as a multi-purpose system.

(3) Effectiveness as the training tool: In this study, with regard to training effect, only visual items were confirmed. We will work on the issue of whether it is possible to improve accuracy in force sense such as loading force by training in the future. 
With regard to the system in this study, we have not described the relation between sampling error and registration error. If error occurs in obtaining the sampling points, errors may occur in position/posture of the femur/tibia coordinate system calculated from the femur/lower leg receivers. With regard to reliability of position/posture information shown by this system, we will describe our findings in another paper.

\section{Acknowledgment}

Part of this study was conducted with assistance from Ministry of Education, Culture, Sports, Science and Technology, Special Coordination Funds for the promotion of Sci. and Tech., Strategic Research Base Program "Formation of Consolidated Research Base for Advanced Science and Medical Care" and Japan Society for the Promotion of Science, Global COE program "Global Robot Academia."

\section{References}

( 1 ) Cipriano, J.J., Photographic Manual of Regional Orthopaedic and Neurologic Tests, (2010), Lippincott Williams and Wilkins.

( 2 ) Reider, B., The Orthopaedic Physical Exam, (2004), Elsevier Saunders.

( 3 ) Harner, C.D., Vince, K.G, Fu, F.H., Knee Surgery, (1994), Williams and Wilkins.

( 4 ) Lerat, J.L., Moyen, B., Jenny, J.Y., Perrier, J.P., “A comparison of pre-operative evaluation of anterior knee laxity by dynamic X-rays and by the arthrometer KT 1000", Knee Surgery, Sports Traumatology, Arthroscopy, Vol.1-No.1, (2005), pp.54-59.

( 5 ) Kowalk, D.L., Wojtys, E.M., Disher, J., Loubert, P., "The relationships between instrumented measurements of ankle and knee ligamentous laxity and generalized joint laxity”, The American Journal of Sports Medicine, Vol.21-No.5, (1993), pp.744-747.

( 6 ) Pearsall, A.W., Kovaleski, J.E., Heitman, R.J., Gurchiek, L.R., Hollis, J.M., "Quantitative analysis of the measuring capabilities of the KT-1000 knee ligament arthrometer", The Journal of Sports Medicine and Physical Fitness, Vol.46-No.1, (1993), pp.104-110.

( 7 ) Makita, S., Uraoka, S., Masutani, Y., Nishimura, A., "Consideration of Torque Model for Knee Joint of Leg Robot for Training of Physical Therapist Students", The JSME Symposium on Welfare Engineering 2005, (2005), pp.221-224.

( 8 ) Kikuchi, T., Oda, K., Yamaguchi, S., Furusho, J., "Development of Leg Robot for Simulation of Spastic Movement with Compact MR Fluid Clutch", Journal of the Robotics Society of Japan, Vol.27-No.8, (2009), pp.933-941.

( 9 ) Morita, Y., Kawai, Y., Ukai, H., Sanaka, K., Nakamuta, H., Takao, K., "Development of Leg Robot for Physical Therapy Training - Proposal of Knee Joint Mechanism with Rolling, Sliding and Coming Off -,", Proceedings of International Conference on Control, Automation and Systems 2010, (2010), pp.151-155.

(10) Vankipuram, M., Kahol, K., McLaren, A., Panchanathan, S., "A virtual reality simulator for orthopedic basic skills: a design and validation study", Journal of Biomedical Informatics, Vol.43-No.5, (2010), pp.661-668.

(11) Frey, M., Hoogen, J., Riener, R., "The 9 DOF Haptic Display of the Munich Knee Simulator: Setup and Experimental Results", Proceedings of EuroHaptics 2004, (2004), pp.395-398.

(12) Riener, R., Frey, M., Proll, T., Regenfelder, F., Burgkart, R., "Phantom-based multimodal interactions for medical education and training: the Munich Knee Joint Simulator", IEEE Transactions on Information Technology in Biomedicine, Vol.8-No.2, (2004), pp.208-216.

(13) Grood, E.S., Suntay, W.J., "A joint coordinate system for the clinical description of three-dimensional motions: Application to the knee", Journal of Biomechanical Engineering, Vol.105-No.2, (1983), pp.136-144.

(14) Hoshino, Y., Kuroda, K., Nagamune, K., Kurosaka, M., "In vivo measurement of the 
pivot-shift test in the anterior cruciate ligament-deficient knee using an electromagnetic device", The American Journal of Sports Medicine, Vol.35-No.7, (2007), pp.1098-1104.

(15) Fujie, H., Kashiwaguchi, S., Glen, L.A., "Magnitude and Distribution of the In Situ Force in the Human Anterior Cruciate Ligament during a Joint Instability Test (Lachman Test)", Transactions of the Japan Society of Mechanical Engineers. A, Vol.60-No.578, (1994), pp.2459-2463.

(16) Markolf, K.L., Mensch, J.S., Amstutz, H.C., "Stiffness and laxity of the knee-the contributions of the supporting structures. A quantitative in vitro study", Journal of Bone Joint Surgery, Vol.58-No.5, (1976), pp.583-594.

(17) Markolf, K.L., Kochan, A., Amstutz, H.C., "Measurement of knee stiffness and laxity in patients with documented absence of the anterior cruciate ligament", Journal of Bone Joint Surgery, Vol.66-No.2, (1984), pp.242-252.

(18) Fujie, H., Watanabe, H., Tsukamoto, Y., Sasada, T., Mabuchi, K., "In-situ Forces in Human Knee Ligaments During an Instability Test : Measurement using Robotics", Transactions of the Japan Society of Mechanical Engineers. C, Vol.61-No.586, (1995), pp.2521-2526.

(19) Kitami, K., Fujie, H., Isao, M., "Use of robotic technology to measure anterior instability of human knee joint : A biomechanical study using amputated knees”, Kitasato medicine, Vol.25-No.3, (1995), pp.259-266.

(20) Takaoka, K., Seikeigeka-Tosyukensahou, (2003), Medical View.

(21) Teghtsoonian, R., "On the exponents in Stevens' law and the constant in Ekman's law", Psychological Review, Vol.78-No.1, (1971), pp.71-80. 